\title{
Probabilistic Correspondence Matching using Random Walk with Restart
}

\author{
Changjae Oh \\ ocj1211@yonsei.ac.kr \\ Bumsub Ham \\ mimo@yonsei.ac.kr \\ Kwanghoon Sohn \\ khsohn@yonsei.ac.kr
}

\author{
School of Electrical and Electronic \\ Engineering \\ Yonsei University \\ Seoul, Korea
}

\begin{abstract}
This paper presents a probabilistic method for correspondence matching with a framework of the random walk with restart (RWR). The matching cost is reformulated as a corresponding probability, which enables the RWR to be utilized for matching the correspondences. There are mainly two advantages in our method. First, the proposed method guarantees the non-trivial steady-state solution of a given initial matching probability due to the restarting term in the RWR. It means the number of iteration, a crucial parameter which influences the performance of algorithm, is not needed in contrast to the conventional methods. This gives the consistent results regardless of the evolution time. Second, only an adjacent neighborhood is considered when the matching probabilities are inferred, which lowers the computational complexity while not sacrificing performance. Experimental results show that the performance of the proposed method is competitive to that of state-of-the-art methods both qualitatively and quantitatively.
\end{abstract}

\section{Introduction}

It is important to find correspondences between images in the field of computer vision, thus lots of solutions have been proposed to address this problem. There are mainly two classes (global methods and local methods) in correspondences matching [四]. Global methods find the corresponding point, a globally optimal solution of an energy functional [ $\square, \mathbf{Q}, \mathbf{\theta}]$, in which the smoothness assumption is explicitly leveraged. Local methods compute correlation between points within a matching window with an assumption that all pixels in a matching window have a similar disparity. This implies that an appropriate size and a shape of the window significantly influence the performance of local methods [ $\mathbf{Q}, \mathbf{\theta}]$. There have been many approaches for alleviating this problem. Fusiello and Roberto proposed the multiwindow method which chooses the best window among pre-defined ones []. Kanade and Okutomi used an adaptive window in order to localize only relevant disparities [0]. However, it cannot localize well at depth discontinuities due to the rectangular window shape, which results in the foreground fatting effect. Yoon and Kweon proposed an adaptive weight method [ $[$ ]. A support weight at each point was computed according to the color and spatial distances between points. Then, the bilateral filtering [] $[$ was performed to aggregate 


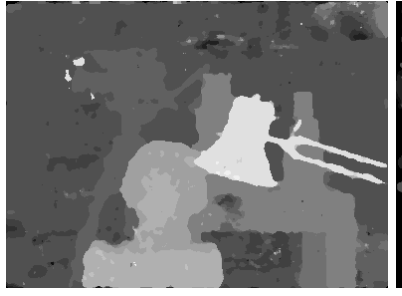

(a) Adaptive weight (AW) [0]

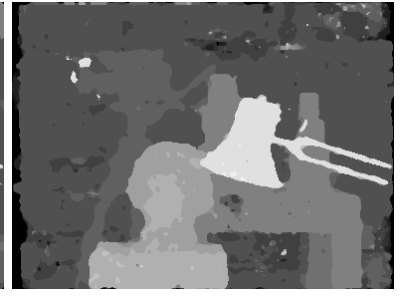

(b) Cost filter (CF) []]

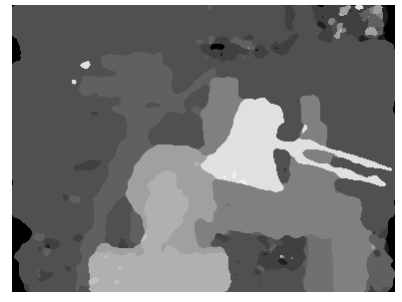

(c) Anisotropic diffusion (AD) [ $[\mathbf{Q}]$

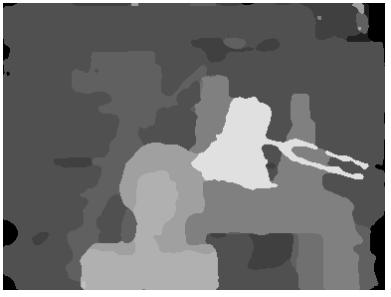

(d) Geodesic diffusion (GD)

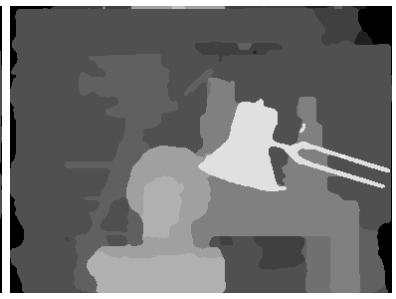

(e) The proposed method

Figure 1: Disparity estimation results with the same neighborhood, i.e., 4-neighborhood, in cost aggregation. (a) Adaptive weight (AW) [0], (b) Cost filter (CF) []] , (c) Anisotropic diffusion (AD) [ $\mathrm{G}]$, (d) Geodesic diffusion (GD) []] and (e) The proposed method. The proposed method gives non-trivial steady-state solution of each matching probability plane, which means there is no need to consider an optimal window size. The percentages of bad matching error of (a)-(e) are 3.43, 3.24, 3.85, 2.96, and 1.97, respectively [ $\mathbb{}$ ] $]$. The comparative computation times of (a)-(e) are 2.87, 1.18, 0.93, 2.19, and 1.0, respectively, when the computation time of the proposed method is normalized to 1.0.

an initial matching cost. The performance of adaptive weight method is comparable to that of other global methods. However, it causes high complexity due to the large window size. Many methods have been proposed to reduce the complexity of adaptive weight method, while not sacrificing high performance $[\mathbf{\theta}, \mathbb{\square}, \mathbb{\square}, \mathbb{\square}, \mathbb{\square}]$. Min and Sohn presented the multi-scale approach in both an image and a cost domain [ $\mathrm{\theta}$ ]. Richardt et al. [四] used the bilateral grid [四] to reduce the complexity of adaptive weight method [0]. Rhemann et al. applied guided filter [ㅁ] , an alternative to the bilateral filter [Q], to cost aggregation by leveraging that it preserves the edges well and computation time is invariant to the window size [] . Recently, De-Maeztu and Villanueva presented a diffusion-based correspondence matching in which the computed weights as well as the costs were diffused so as to lower the computational cost []. While most methods have concentrated on reducing the image resolution and on modifying the window size and/or the shape, Min et al. accelerated the matching speed by compressing a search range space and sampling points in the matching window [ए]].

In this paper, we propose a probabilistic method for correspondence matching with a framework of the random walk with restart (RWR). The matching cost is viewed of as a probability of being matched between points, which enables the RWR to be utilized for matching the correspondences. The significance of our work is as follows. First, only an adjacent neighborhood is needed to infer the matching probability, which reduces the complexity of algorithm. Second, the proposed method gives steady-state distribution of each matching probability, and it could be an alternative to other existing optimization techniques 
such as dynamic programming [ []], graph cuts [ $[$ ] $]$, and belief propagation [ $⿴ 囗 十)$. Figure 1 shows that the performance of the proposed method is superior to that of state-of-the-art methods within a same configuration.

There was an attempt to apply the random walk (RW) theory to the correspondence matching. Shen et al. inferred the disparity map by finding a minimum of a given energy functional via the RW [ $\square, \square]$. However, the RW framework does not give a meaningful solution in a steady-state as will be explained later, which results in a noisy result in textureless region.

The remainder of this paper is organized as follows. In section 2, we formalize the matching problem as a probability inferring process and describe the proposed method in section 3. Experimental results are presented in section 4. Finally, we conclude the paper in section 5 .

\section{Motivation}

Local methods are mainly composed of three steps [四]: matching cost computation, cost aggregation, and disparity computation. Let us assume that stereo images are rectified and a truncated absolute difference (TAD) is used to compute the matching cost. Then, each step is represented as follows:

$$
\begin{aligned}
\text { Matching cost computation: } & e_{0}(\mathbf{x}, \mathbf{d})=\min \left(\left\|I_{R}(\mathbf{x})-I_{T}(\mathbf{x}, \mathbf{d})\right\|_{1}, \sigma\right) \\
\text { Cost aggregation: } & e_{n+1}(\mathbf{x}, \mathbf{d})=\frac{\sum_{\mathbf{y} \in \mathcal{N}} w(\mathbf{x}, \mathbf{y}) e_{n}(\mathbf{y}, \mathbf{d})}{\sum_{\mathbf{y} \in \mathcal{N}} w(\mathbf{x}, \mathbf{y})} \\
\text { Disparity computation: } & \mathbf{d}(\mathbf{x})=\underset{\mathbf{d} \in\left[\mathbf{d}_{1}, \ldots, \mathbf{d}_{D}\right]}{\arg \min } e_{N}(\mathbf{x}, \mathbf{d})
\end{aligned}
$$

where $\mathbf{x}=[x, y]^{T}$ and $\mathbf{d}=[d, 0]^{T}$ represent the position and disparity vector, respectively. The weight between $\mathbf{x}$ and $\mathbf{y}$ is defined as $w(\mathbf{x}, \mathbf{y})$. First, an initial matching cost $e_{0}(\mathbf{x}, \mathbf{d})$ is computed by an absolute difference between points on the reference image $I_{R}(\mathbf{x})$ and on the 'd'-shifted target image $I_{T}(\mathbf{x}, \mathbf{d})$ with the threshold $\sigma$ as in Equation 1. Then, in Equation 2, the cost $e_{n}(\mathbf{x}, \mathbf{d})$ is iteratively aggregated with $\mathbf{y} \in \mathcal{N}$ where $\mathcal{N}$ is the neighborhood of $\mathbf{x}$. Finally, an optimal disparity $\mathbf{d}(\mathbf{x})$ is selected within a search range $D$ for the aggregated cost $e_{N}(\mathbf{x}, \mathbf{d})$ after the maximum iteration $N$, by winner-takes-all (WTA) as in Equation 3.

This procedure can be re-formulated as follows []]:

Matching probability computation: $\quad p_{0}(\mathbf{x}, \mathbf{d})=\max \left(\sigma-\left\|I_{R}(\mathbf{x})-I_{T}(\mathbf{x}, \mathbf{d})\right\|_{1}, 0\right)$

$$
\begin{array}{cl}
\text { Probability inference: } & p_{n+1}(\mathbf{x}, \mathbf{d})=\frac{\sum_{\mathbf{y} \in \mathcal{N}} w(\mathbf{x}, \mathbf{y}) p_{n}(\mathbf{y}, \mathbf{d})}{\sum_{\mathbf{y} \in \mathcal{N}} w(\mathbf{x}, \mathbf{y})} \\
\text { Disparity computation: } & \mathbf{d}(\mathbf{x})=\underset{\mathbf{d} \in\left[\mathbf{d}_{1}, \ldots, \mathbf{d}_{D}\right]}{\arg \max } p_{N}(\mathbf{x}, \mathbf{d})
\end{array}
$$

Then, the matching cost $e_{0}(\mathbf{x}, \mathbf{d})$ can then be regarded as a likelihood $p_{0}(\mathbf{x}, \mathbf{d})$ of being matched between points as in Equation 4, i.e., a likelihood $p_{0}(\mathbf{x}, \mathbf{d})$ is a probability that the corresponding point of reference pixel $\mathbf{x}$ is the ' $\mathbf{d}$ '-shifted point of target image, and is inversely proportional to the matching cost $e_{0}(\mathbf{x}, \mathbf{d})$. Accordingly, aggregating the cost $e_{n+1}(\mathbf{x}, \mathbf{d})$ with an initial matching cost $e_{0}(\mathbf{x}, \mathbf{d})$ can be re-formulated as inferring the probability $p_{n+1}(\mathbf{x}, \mathbf{d})$ with an initial matching probability $p_{0}(\mathbf{x}, \mathbf{d})$. 


\section{Probabilistic Correspondence Matching with RWR}

\subsection{Problem statement}

In section 2, a correspondence matching problem was formulated as a probability inferring problem. The object is then to find an optimal matching probability $p_{N}(\mathbf{x}, \mathbf{d})$ for a given initial matching probability $p_{0}(\mathbf{x}, \mathbf{d})$.

The RW theory has been widely used to optimize probabilistic problems [ㅁ, $\square, \mathbb{\square}, \mathbb{\square}$ ]. It has been known that the RW and the Laplace equation give the same solution, which means that the steady-state of a given energy functional can be captured by the RW [ㅁ] ]. However, the steady-state solution of the RW gives no meaningful information. Formally, the right eigenvector corresponding to the largest eigenvalue of $\overline{\mathbf{W}}$ in Equation 9 is constant, i.e., the steady-state solution of the RW is a constant signal.

Let us consider a problem of inferring an optimal probability as in Equation 5. Inferring a probability with a small neighborhood, i.e., 4-neighborhood, is the same as a procedure of the RW [ㅁ], which means the adaptive weight method, a variation of Equation 2 or Equation 5, does not provide a meaningful steady-state solution similar to the RW. Note that Equation 2 becomes the multi-window method [0], the adaptive window method [ $[$ ], and the adaptive weight method [0] according to the weight function $w(\mathbf{x}, \mathbf{y})$ and/or the neighborhood $\mathcal{N}$. Thus, in conventional methods, the number of iteration should be specified in advance and it significantly influences the performance of algorithms [ $\mathbf{\square}, \mathbf{Q}, \mathbf{\square}, \mathbf{\square}, \mathbf{\square}]$. Recently, the RWR has become increasingly popular, since its restarting term gives the meaningful information in a steady-state, allowing it to consider the global relation at all scales [ष] ]. Therefore, we infer the optimal matching probability $p_{N}(\mathbf{x}, \mathbf{d})$ via the RWR in contrast to the conventional methods which are based on the RW such as the adaptive weight [ $[0]$ and the diffusion based method [ $[\mathbb{Z}]$. Note that the RW and the anisotropic diffusion are also closely related to each other [एष].

\subsection{Graph model}

Consider an initial matching probability as an undirected graph $G=(V, E)$ with nodes $V$ and edges $E$. Each node $v_{i} \in V$ indicates a point at $\mathbf{x}_{i} \in\left\{\mathbf{x}_{1}, \ldots, \mathbf{x}_{M}\right\}$ in an initial matching probability where $M$ is the size of reference image. The adjacent nodes $v_{i}$ and $v_{j}$ are connected to an edge $e_{i j} \in E$. The graph assigns a weight to each edge and the weight is computed in the reference image. With an assumption that neighboring pixels tend to have similar disparity values when their color distance is small, the edge weight is computed as follows:

$$
w_{i j}=\exp \left(-\frac{\left\|I_{R}\left(\mathbf{x}_{i}\right)-I_{R}\left(\mathbf{x}_{j}\right)\right\|_{2}^{2}}{\gamma_{c}}\right)
$$

where $\gamma_{c}$ represents the color variance. $I_{R}\left(\mathbf{x}_{i}\right)$ and $I_{R}\left(\mathbf{x}_{j}\right)$ represent an intensity of reference image at $\mathbf{x}_{i}$ and $\mathbf{x}_{j}$ in $L a b$ color space, respectively.

\subsection{Probability inference}

With a weighted graph, a probability in a steady-state can be inferred via the RWR. A random walker, with an initial position $\mathbf{x}_{j}$, iteratively transits to its neighboring points according to the edge weight as in Equation 7 until it reaches to the reference position $\mathbf{x}_{i}$. Also, the 
random walker goes back to the initial position $\mathbf{x}_{j}$ with the restarting probability at each iteration. The RWR is formulated in an iterative manner as follows [ $[\mathrm{Z}]$ :

$$
\begin{aligned}
\mathbf{P}_{n+1}^{k} & =(1-\alpha) \mathbf{D}^{-1} \mathbf{W} \mathbf{P}_{n}^{k}+\alpha \mathbf{P}_{0}^{k} \\
& =(1-\alpha) \overline{\mathbf{W}} \mathbf{P}_{n}^{k}+\alpha \mathbf{P}_{0}^{k}
\end{aligned}
$$

where $\mathbf{P}_{n}^{k}=\left[p_{n}\left(\mathbf{x}_{i}, \mathbf{d}_{k}\right)\right]_{M \times 1}$ denotes an initial matching probability when the number of iteration is $n$. The restarting probability is represented as $\alpha$. The adjacency matrix $\mathbf{W}=\left[w_{i j}\right]_{M \times M}$ is normalized as $\overline{\mathbf{W}}=\mathbf{D}^{-1} \mathbf{W}$, where $\mathbf{D}=\operatorname{diag}\left(D_{1}, \ldots, D_{M}\right)$, and $D_{i}=\sum_{j=1}^{M} w_{i j}$. When the solution reaches to the steady-state, $\mathbf{P}_{n}^{k}$ and $\mathbf{P}_{n+1}^{k}$ become identical, i.e., the energy transition with respect to time approaches 0 . Therefore, Equation 8 can be reformulated as follows:

$$
\begin{aligned}
\mathbf{P}_{s}^{k} & =(1-\alpha) \overline{\mathbf{W}} \mathbf{P}_{s}^{k}+\alpha \mathbf{P}_{0}^{k} \\
& =\alpha(I-(1-\alpha) \overline{\mathbf{W}})^{-1} \mathbf{P}_{0}^{k} \\
& =\mathbf{R} \mathbf{P}_{0}^{k}
\end{aligned}
$$

where $\mathbf{P}_{s}^{k}$ is a matching probability in a steady-state. $\mathbf{R}$ can be interpreted as affinity scores between points in an initial state $\mathbf{P}_{0}^{k}$. We can see that all paths are considered when $\mathbf{P}_{s}^{k}$ is computed. Furthermore, $\mathbf{R}$ can be re-formulated as an infinite geometric series:

$$
\begin{aligned}
\mathbf{R} & =\alpha(I-(1-\alpha) \overline{\mathbf{W}})^{-1} \\
& =\alpha \sum_{n=0}^{\infty}(1-\alpha)^{n} \overline{\mathbf{W}}^{n}
\end{aligned}
$$

That is, $\mathbf{R}$ is a weighted sum of $\overline{\mathbf{W}}^{n}$, whose element $\bar{w}_{i j}^{n}$ is a probability that a random walker transits $\mathbf{x}_{j}$ to $\mathbf{x}_{i}$ after $n$ iterations. The iteration $n$ denotes a scale of the transition, e.g., the random walker transits farther as $n$ becomes larger [ष्व]. Accordingly, the RWR optimizes an initial matching probability by considering all paths between two points at all scales. It might seem that the complexity of the proposed method is high since the matrix inversion requires $O\left(M^{3}\right)$ operation. However, we use only an adjacent neighborhood, i.e., 4-neighborhood, which makes $\mathbf{R}$ sparse. Thus, the matrix inversion can be computed with low complexity.

With a steady-state probability, a disparity can be simply selected by WTA strategy as follows:

$$
\mathbf{d}\left(\mathbf{x}_{i}\right)=\underset{\mathbf{d}_{k}}{\arg \max } p_{s}\left(\mathbf{x}_{i}, \mathbf{d}_{\mathbf{k}}\right)
$$

where $p_{s}\left(\mathbf{x}_{i}, \mathbf{d}_{\mathbf{k}}\right)$ is an optimized steady-state probability obtained in Equation 9, and $\mathbf{d}_{k} \in$ $\left\{\mathbf{d}_{1}, \ldots, \mathbf{d}_{D}\right\}$.

The correspondence matching within the RWR framework has the following advantages: 1) A non-trivial steady-state solution is guaranteed by constraining a steady-state probability to an initial matching probability to some extent, which means that it is not needed to specify the number of iteration. In conventional methods, it is crucial to specify the number of iteration since the performance and the computation time largely depends on this parameter $[\mathbf{Q}, \mathbb{\square}]$. 2) The global relationship between points or the steady-state solution can be captured by using an adjacent neighborhood only, which lowers the complexity of algorithm while maintaining the performance. Accordingly, the proposed method gives high quality matching performance in a semi-global manner with low complexity. 


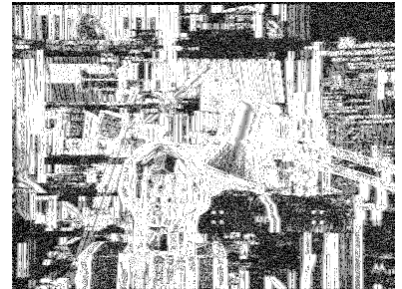

(a) Initial probability $p_{0}(\mathbf{x}, \mathbf{0})$

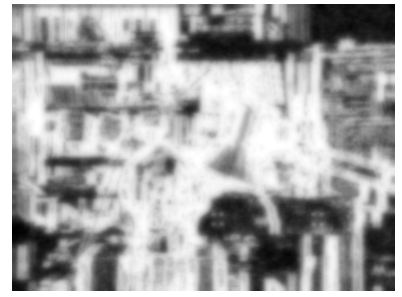

(d) Anisotropic diffusion (AD) [B]

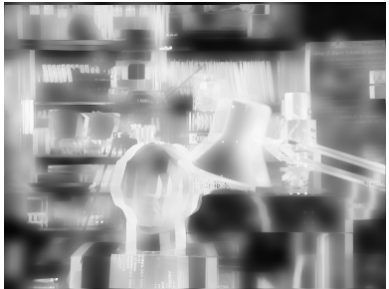

(b) Adaptive weight (AW) [ $[$ ]

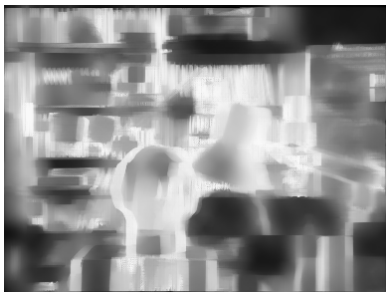

(e) Geodesic diffusion (GD) [ロ]

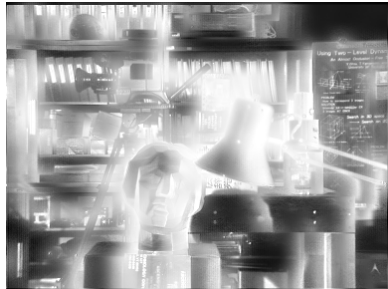

(c) Cost filter (CF) [D]

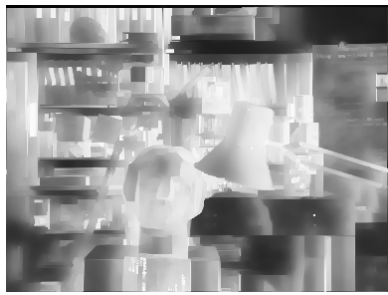

(f) The proposed method

Figure 2: An inferred matching probability in 'Tsukuba' image when the candidate of disparity is 0 . (a) An initial matching probability $p_{0}(\mathbf{x}, \mathbf{0})$, (b) Adaptive weight (AW) [ $\mathbf{\square}$, (b)

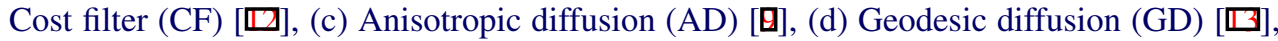
and (e) The proposed method. The low intensity indicates high probability of being matched, and vice versa.

\section{Experimental Results}

In order to verify the performance, the proposed method was compared with state-of-the-

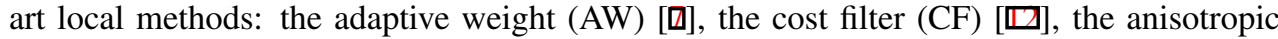
diffusion (AD) $[\mathrm{G}]$, and the geodesic diffusion (GD) [ $[\mathbf{\square}]$. Note that the AD is not a main proposal of [ $[\theta]$, but just a part of their method. We used a TAD of color image $I_{R}$ and $I_{T}$, and a gradient value of gray image $I_{R}{ }^{\prime}$ and $I_{T}{ }^{\prime}$ as an initial matching probability as follows:

$$
\begin{aligned}
p_{0}(\mathbf{x}, \mathbf{d}) & =\lambda \cdot \max \left(\sigma_{1}-\left\|I_{R}(\mathbf{x})-I_{T}(\mathbf{x}, \mathbf{d})\right\|_{1}, 0\right) \\
& +(1-\lambda) \cdot \max \left(\sigma_{2}-\left\|\nabla_{x} I_{R}(\mathbf{x})-\nabla_{x} I_{T}{ }^{\prime}(\mathbf{x}, \mathbf{d})\right\|_{1}, 0\right)
\end{aligned}
$$

where $\nabla_{x}$ denotes the gradient operator along a horizontal direction. $\lambda$ controls the leverage between a color and a gradient term. It was set to 0.11 and truncation values $\sigma_{1}$ and $\sigma_{2}$ were set to 15 and 2, respectively. For the post processing, left-right consistency check and a weighted median filter were applied [ $\square]$.

Since the performance of conventional methods largely depend on the window size and parameters, they were carefully set through intensive experiments. The window size of the AW and CF was set to $35 \times 35$ and $19 \times 19$, respectively. The AD, GD and the proposed method used 4-neighborhood. For the AD, the number of iteration was set to 100 with the step size being 0.01 . In the GD, the number of iteration was set to 18 . In our algorithm, the color variance $\gamma_{c}$ and the restarting probability $\alpha$ was set to 50 and 0.003 , respectively. The results of the CF were simulated with a source code provided by the author [ $\square$ ].

Figure 2 shows an initial matching probability $p_{0}(\mathbf{x}, \mathbf{0})$ and an inferred probability map when the candidate of disparity is 0 . The AW shows a higher performance than the CF 


\begin{tabular}{|c|c|c|c|c|c|c|c|c|c|c|c|c|}
\hline \multirow[t]{2}{*}{ Algorithm } & \multicolumn{3}{|c|}{ Tsukuba } & \multicolumn{3}{|c|}{ Venus } & \multicolumn{3}{|c|}{ Teddy } & \multicolumn{3}{|c|}{ Cones } \\
\hline & nonocc & all & disc & nonocc & all & disc & nonocc & all & disc & nonocc & all & disc \\
\hline AW [D] & 2.43 & 2.77 & 11.6 & 0.24 & 0.46 & 2.45 & 7.90 & 13.2 & 17.8 & 3.36 & 8.60 & 8.29 \\
\hline AW [D]* & 1.38 & 1.85 & 6.90 & 0.71 & 1.19 & 6.13 & 7.88 & 13.3 & 18.6 & 3.97 & 9.79 & 8.26 \\
\hline CF [四] & 1.76 & 2.14 & 8.34 & 0.19 & 0.46 & 2.51 & 6.24 & 11.5 & 16.0 & 2.48 & 8.01 & 7.20 \\
\hline CF [四]* & 1.51 & 1.85 & 7.61 & 0.20 & 0.39 & 2.42 & 6.16 & 11.8 & 16.0 & 2.71 & 8.24 & 7.66 \\
\hline AD [日] & 2.93 & 3.85 & 11.6 & 1.09 & 1.78 & 11.9 & 8.78 & 14.2 & 19.9 & 3.19 & 8.83 & 9.13 \\
\hline GD [ㅁ] & 2.39 & 2.96 & 11.5 & 0.25 & 0.45 & 3.31 & 7.28 & 12.4 & 17.7 & 3.12 & 8.65 & 8.98 \\
\hline GD [四]* & 1.88 & 2.35 & 7.64 & 0.38 & 0.82 & 3.02 & 5.99 & 11.3 & 13.3 & 2.84 & 8.33 & 8.09 \\
\hline Proposed method & 1.60 & 1.97 & 6.44 & 0.20 & 0.38 & 2.51 & 6.15 & 11.5 & 15.8 & 2.60 & 7.92 & 7.48 \\
\hline
\end{tabular}

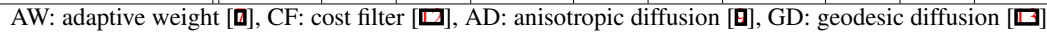

Table 1: Object evaluation for the proposed method

in edge-preserve smoothing. However, in both methods, a non-smoothed texture region is observed, which results in a noisy disparity map. The GD shows better performance than the $\mathrm{AD}$, and localizes the probability map well. However, both methods do not preserve the boundary, which causes the artifacts at depth discontinuities. In our method, a texture region is effectively smoothed as well as the boundary is well-preserved.

Figure 3 compares the performance of the proposed method with that of state-of-theart methods in Middlebury test bed [四]: 'Tsukuba', 'Venus', 'Teddy', and 'Cones'. Even though the proposed method uses a 4-neighborhood in inferring the matching probability, high quality disparity maps are provided which are competitive to other methods. Also, the results of the proposed method show high performance at depth boundaries. For an object comparison, we evaluated the proposed method with respect to the three criteria: non-occluded region (nonocc), all region (all), and discontinuous region (disc) as shown in Table 1. The symbol '*' indicates the results at the Middlebury test bed [四]. It shows that the proposed method shows competitive results with state-of-the-art methods. Noting that disc criterion evaluates the performance only at depth discontinuities, we can see a coherent results with a boundary preserving capacity of the proposed method and other methods as shown in Figure 2 and Figure 3. The comparison of a computation time is shown in Figure 4a. Note that the computation time of the proposed method was normalized to 1.0. Due to the large window size, the AW shows the slowest computation time. The AD shows the fastest results, but its performance is quiet inferior to other methods. In spite of matrix inversion, the proposed method ranks second since the matrix is sparse. In conclusion, the proposed method shows competitiveness to state-of-the art methods both qualitatively and quantitatively. In order to compare the performance in case of a similar window size, we conducted another experiment by changing a window size of the AW and $\mathrm{CF}$ to $3 \times 3$ which is the similar to that used in the $\mathrm{AD}, \mathrm{GD}$ and the proposed method, i.e., 4-neighborhood. Figure 1a and Figure 1b show the results of the AW and CF, which implies that the results of these methods heavily depend on the window size. Figure $4 \mathrm{~b}$ shows the corresponding computation time. It shows that the AW becomes faster since the small window was used. Note that the computation time of the CF does not change since only $O(1)$ operation is needed in the guided filter $[\mathbb{} \mathbf{D}]$. However, its performance heavily depends on the window size by comparing the result in Figure $1 \mathrm{~b}$ with that in Figure 3d.

In our approach, the restarting probability $\alpha$ is an important parameter since it is related to the degree of smoothing of an initial matching probability. The results obtained by changing $\alpha$ are shown in Figure 5. More smoothed result is shown as $\alpha$ becomes smaller, which implies that a random walker rarely returns to the initial position. As we mentioned before, the solution becomes trivial (i.e. a grey image) if the restarting probability approaches to 0 , i.e. the RW, as shown in Figure 5d. 

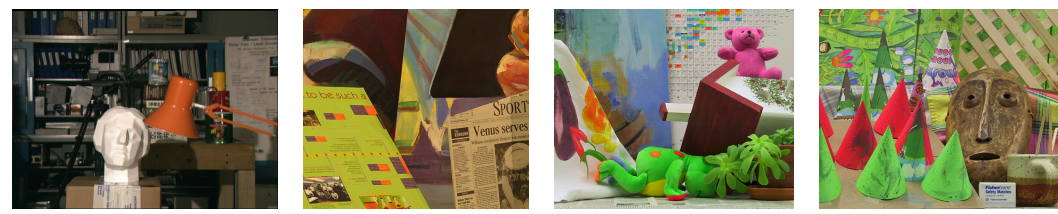

(a) Reference image
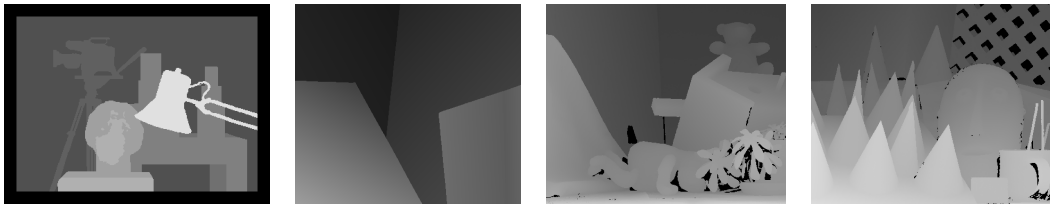

(b) Ground truth
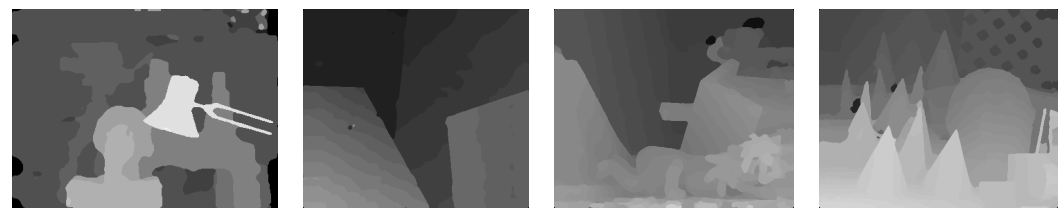

(c) Adaptive weight (AW) [ []
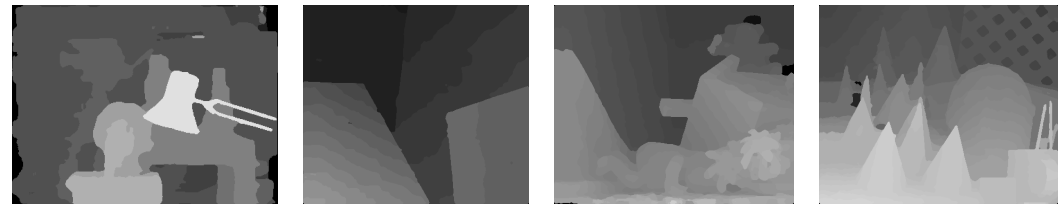

(d) Cost filter (CF) []
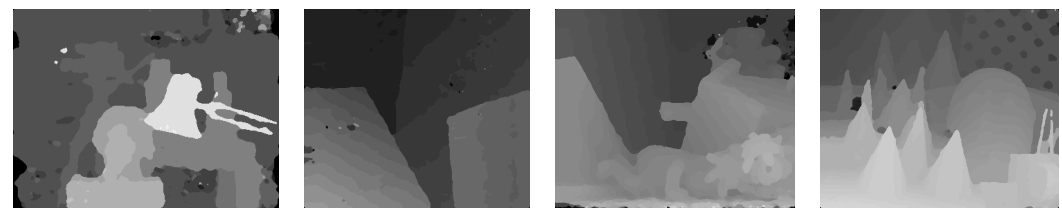

(e) Anisotropic diffusion (AD) [
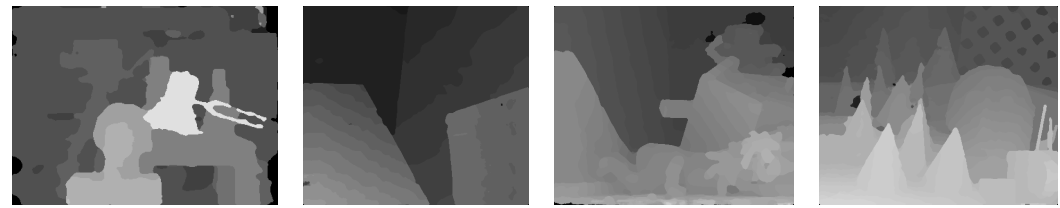

(f) Geodesic diffusion (GD) [ㅁ]
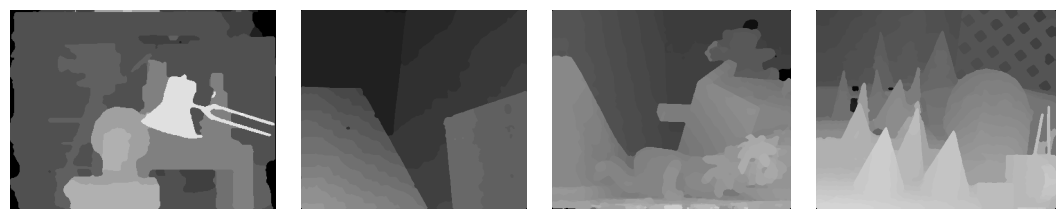

(g) The proposed method

Figure 3: Results for (from left to right) 'Tsukuba', 'Venus', 'Teddy', and 'Cones'. (a) Reference image, (b) Ground truth, (c) Adaptive weight (AW) [Q], (f) Cost filter (CF) [] ], (d) Anisotropic diffusion (AD) [ $[$ ] , (e) Geodesic diffusion (GD) [ $\square]$, and (g) The proposed method. 


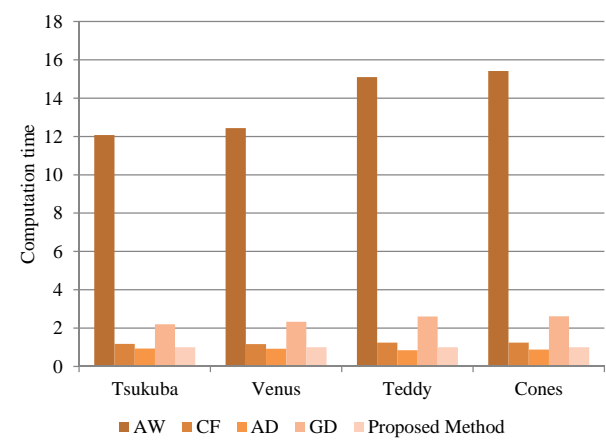

(a)

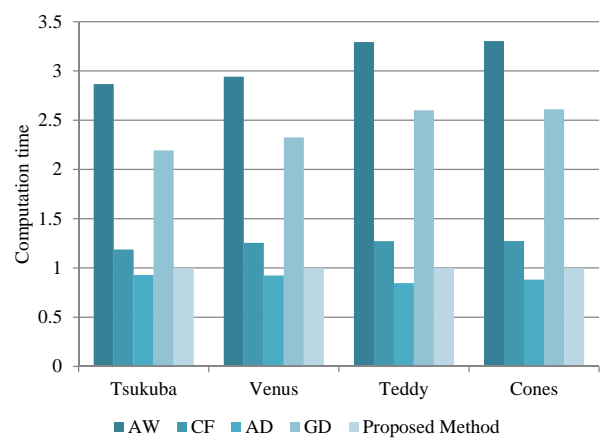

(b)

Figure 4: The computation time of disparity estimation with (a) an optimal window and (b) the smallest window. Note that the computation time of the proposed method is normalized to 1.0 .

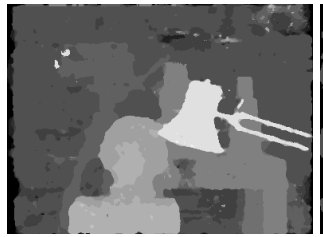

(a)

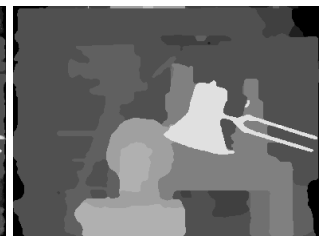

(b)

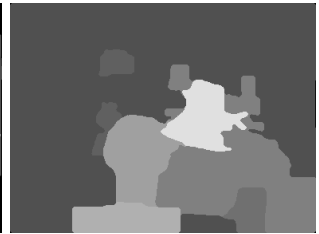

(c)

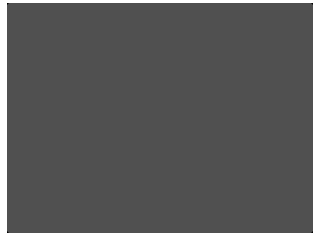

(d)

Figure 5: Disparity estimation results of the proposed method when the restarting probability $\alpha$ is (a) $3 \times 10^{-1}$, (b) $3 \times 10^{-3}$, (c) $3 \times 10^{-5}$, and (d) $3 \times 10^{-7}$.

\section{Conclusion}

We have presented a probabilistic method for correspondence matching via the RWR framework by formulating the cost aggregation as a probability inferring problem. It gives the non-trivial steady-state solution with leveraging an adjacent neighborhood only. Therefore, the proposed method shows high performance with low complexity. In order to verify our approach, the accuracy of matching results and computation time are evaluated. We will accelerate the proposed method further by applying a fast solver for calculating the matrix inversion. Furthermore, since the matching probability of each candidate of disparity is inferred in an independent manner, it is feasible that the proposed method can be accelerated by utilizing this parallel architecture via GPU.

\section{References}

[1] D. Scharstein and R. Szeliski, "A taxanomy and evaluation of dense two-frame stereo correspondence algorithms," International Journal of Computer Vision, 47(1-3):7-42, April 2002.

[2] Y. Ohta and T. Kanade, "Stereo by intra- and inter-scanline search using dynamic programming," IEEE Transactions on Pattern Analysis and Machine Intelligence, 7(2):139-154, March 1985.

[3] Y. Boykov, O. Veksler, and R. Zabih, "Fast approximate energy minimization via graph cuts," IEEE Transactions on Pattern Analysis and Machine Intelligence, 23(11):1222-1239, November 2001. 
[4] P. F. Felzenszwalb and D. P. Huttenlocher, "Efficient belief propagation for early vision," International Journal of Computer Vision, 70(1):41-54, October 2006.

[5] A. Fusiello, V. Roberto, and E. Trucco, "Efficient stereo with multiple windowing," in Proc. IEEE Conference on Computer Vision and Pattern Recognition, pages 858-863, 1997.

[6] T. Kanade and M. Okutomi, "Stereo matching algorithm with an adaptive window: Theory and experiment," IEEE Transactions on Pattern Analysis and Machine Intelligence, 16(9):920-932, September 1994.

[7] K. Yoon and I. Kweon, "Adaptive support-weight approach for correspondence search," IEEE Transactions on Pattern Analysis and Machine Intelligence, 28(4):650-656, April 2006.

[8] C. Tomasi and R. Manduchi, "Bilateral Filtering for Gray and Color Images," in Proc. IEEE International Conference on Computer Vision, pp.839-846, 1998.

[9] D. Min and K. Sohn, "Cost aggregation and occlusion handling with WLS in stereo matching," IEEE Transactions on Image Processing, 17(8):1431-1442, August 2008.

[10] C. Richardt, D. Orr, I. Davies, A. Criminisi, and N. A. Dodgson, "Real-time spatiotemporal stereo matching using the dual-cross-bilateral grid," in Proc. European Conference on Computer Vision, pages 510-523, 2010.

[11] D. Min, J. Lu, and M. N. Do "A revisit to cost aggregation in stereo matching: How far can we reduce its computational redundancy?," in Proc. International Conference on Computer Vision, pages 1567-1574, 2011.

[12] C. Rhemann, A. Hosni, M. Bleyer, C. Rother, and M. Gelautz, "Fast cost-volume filtering for visual correspondence and beyond," in Proc. IEEE Conference on Computer Vision and Pattern Recognition, pages 3017-3024, 2011.

[13] L. De-Maeztu, A. Villanueva, and R. Cabeza, "Near Real-Time Stereo Matching Using Geodesic Diffusion," IEEE Transactions on Pattern Analysis and Machine Intelligence, 34(2):410-416, February 2012.

[14] S. Paris and F. Durand, "Fast approximation of the bilateral filter using a signal processing approach," in Proc. European Conference on Computer Vision, pages 568-580, 2006.

[15] K. He, J. Sun, and X. Tang, "Guided image filtering," in Proc. European Conference on Computer Vision, pages 1-14, 2010.

[16] R. Shen, I. Cheng, X. Li, and A. Basu, "Stereo matching using random walks," In IEEE International Conference on Pattern Recognition, pages 1-4, 2008.

[17] L. Grady, "Multilabel random walker image segmentation using prior models," in Proc. IEEE Conference on Computer Vision and Pattern Recognition, pages 763-770, 2005.

[18] L. Grady, "Random walks for image segmentation," IEEE Transactions on Pattern Analysis and Machine Intelligence, 28(11):1768-1783, November 2006.

[19] T. Kim, K. Lee, and S. Lee, "Generative Image Segmentation Using Random Walks with Restart," in Proc. European Conference on Computer Vision, pages 264-275, 2008.

[20] J. Y. Pan, H. J. Yang, C. Faloutsos, and P. Duygulu, "Automatic multimedia cross-modal correlation discovery," in ACM SIGKDD international conference on Knowledge discovery and data mining, pages 653-658, 2004 